\title{
THE MORPHOLOGY-DENSITY RELATION FOR GALAXIES IN A COLD DARK
} MATTER-DOMINATED UNIVERSE

\author{
A. E. Evrard and J. Silk \\ Department of Astronomy and Center for Particle Astrophysics, University of California, Berkeley \\ AND \\ A. S. Szalay \\ Department of Physics and Astronomy, Johns Hopkins University; and Department of Physics, Eötvös University, Budapest \\ Received 1990 February 12; accepted 1990 June 8
}

\begin{abstract}
We explore a model in which the observed galaxy morphology-density relation is intrinsically related to the initial fluctuations from which galaxies formed in a universe dominated by cold dark matter (CDM). We assume a direct mapping between initial peak height and final galaxy morphology with sharp thresholds dividing the principal Hubble types. Using a simplified, fully analytic formalism appropriate to Gaussian models, we show how a background cluster or group of galaxies may modulate the abundances of different morphological types. The model accounts for the observed power-law relation between the elliptical fraction and the combined fraction of elliptical and lenticular galaxies. We then extend this analytic approach using $N$-body simulations. The results support the validity of our prescription for differentiating disk from spheroidal galaxies. We note that a power-law segregation between luminous and dark matter densities is predicted by the simulations.
\end{abstract}

Subject headings: cosmology — dark matter — galaxies: clustering — galaxies: structure

\section{INTRODUCTION}

It is well known (Abell 1958; Morgan 1961) that elliptical galaxies are preferentially found in dense regions, such as cluster cores, whereas spirals predominate in the field. Davis and Geller (1976) first provided a quantitative measure of morphological separation in three dimensions by evaluating the respective correlation functions. They found significant differences between the clustering properties of the various morphological types, with ellipticals having a steeper two-point correlation function of enhanced amplitude relative to the spirals, and to the mean of all galaxies in the surveyed volume. This result was extended to a much broader range of densities by Dressler (1980) who computed the morphological type dependence on local density as measured by taking the 10 nearest projected neighbors to each galaxy in a sample which included 55 clusters. Postman and Geller (1984) reevaluated the morphology-density dependence using three-dimensional overdensities extending over a remarkable range of six decades in density. More recently, Giovanelli, Haynes, and Chincarini (1986) analyzed a part of the UGC Catalog to study the morphology-projected density dependence in the PerseusPisces Supercluster and found similar results over a wide range of densities. These results have been especially intriguing to theorists, because they may be indicative of environmental effects during the galaxy formation process, in particular local density, which influence morphological type. For example, galaxy mergers provide an attractive method for forming ellipticals (Toomre and Toomre 1972; Schweizer 1989). However, an alternative model for galaxy formation in a cold dark matter (CDM) universe relies exclusively on initial conditions that characterize the primordial spectrum of density fluctuations laid down in the very early universe from which all nonlinear structure is generated; Faber (1982) and Blumenthal et al. (1984) identify ellipticals with $>3 \sigma$ and spirals with 2-3 $\sigma$ peaks in the primordial Gaussian density fluctuation field. This model, when coupled with a mechanism to distinguish dwarfs from bright galaxies (Dekel and Silk 1986), is capable of reproducing several fundamental characteristics of the observed distribution of bright galaxies (Evrard 1989).

The issue that we address in this paper is whether the observed galaxy morphology-density relation could arise solely from processes intrinsic to the primordial fluctuations from which the galaxies condensed, without the need of any environmentally induced physical processes such as ram pressure stripping. We will focus on the distinction between ellipticals and spirals and consider the possibility of regarding S0's as an intermediate type.

Our procedure will be to commence with the biased CDM hypothesis and explore its consequences for the morphologydensity relation, both with analytic techniques and with $N$-body simulations. In $\S$ II, we describe via the analytic theory of Gaussian fields developed by Bardeen et al. (1986, hereafter BBKS) how modulation by a background field can affect the typical fluctuation on a galaxy scale. We define ellipticals and spirals as approximately $3 \sigma$ and $2 \sigma$ peaks on galaxy scales, tuning the thresholds to explain the finer details of the morphology-density relation. In $\S$ III, we show that three independent data sets provide strong evidence for a morphology threshold model acting on a single underlying population of galactic halos.

A nonlinear, analytic model is developed in $\S$ IV by using a fit to the fractional overdensity in the CfA group catalog. This enables a mapping from linear Gaussian overdensity into a nonlinear overdensity containing the same mass fraction. We then use $N$-body experiments as an independent test of the model in the nonlinear regime. The final section summarizes cur results.

\section{ANALYTIC THEORY}

We will use the techniques developed by BBKS in $\S \mathrm{V} c$ to describe the modulation effects of a background cluster or 


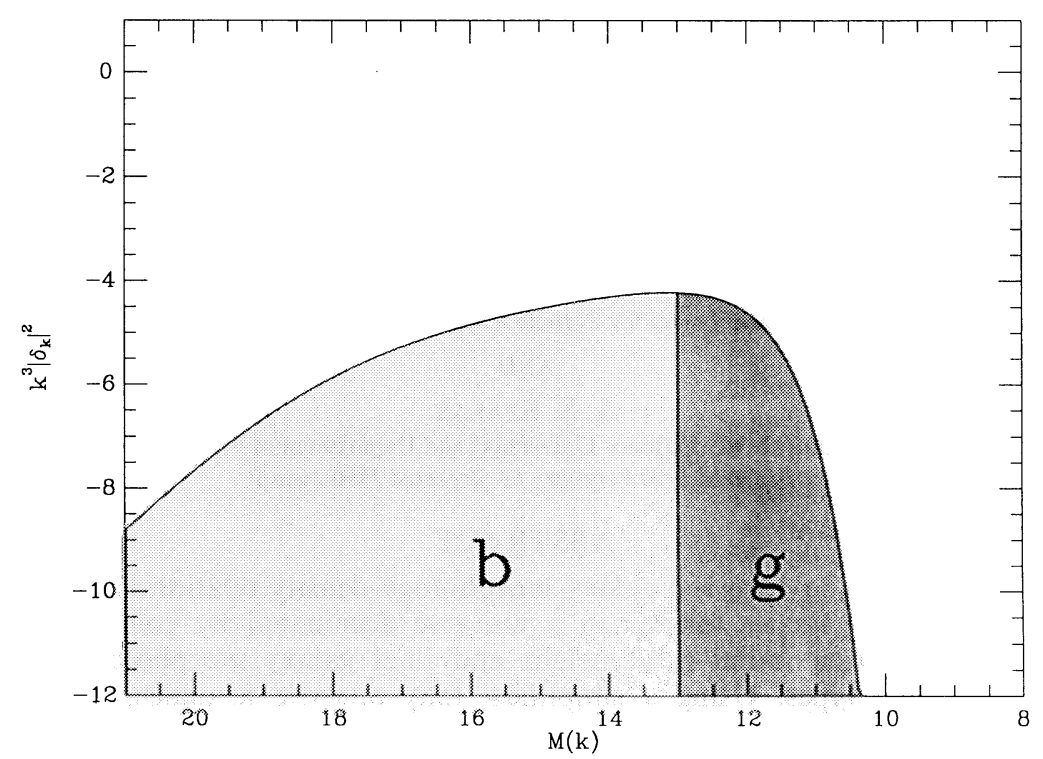

FIG. 1.-The density fluctuation power spectrum of a CDM-dominated universe, with the galaxy-background split at a comoving wavelength $3 h^{-1} \mathrm{Mpc}$. For the spectrum we assume $h=0.5$, and $\Omega=1$.

group on a forming galaxy. We utilize the CDM fluctuation spectrum $P(k)$ given in BBKS. We divide the fluctuations into galactic $\delta_{g}=v_{g} \sigma_{g}$ and large-scale (such as cluster) background $\delta_{b}=v_{b} \sigma_{b}$ components by assuming a sharp split in the fluctuation spectrum. Here $\sigma_{g}$ and $\sigma_{b}$ are the respective rms fluctuations in the two domains and $\delta_{g}, \delta_{b}$ are the corresponding dimensionless overdensities. This $\mathrm{S} / \mathrm{N}$ (signal-to-noise ratio) split is quite common in handling noisy electric signals and assumes that the high-frequency component is uncorrelated with the low-frequency fluctuations. This is justified by the fact that a typical background peak contains such a large number of high-frequency fluctuations that it provides a fair sample. It is the subsequent nonlinearity of biasing in the galaxy formation process that induces the correlations between the low and high frequencies.

Let us describe this mechanism with a simple toy model (Fig. 1). The high-frequency field $v_{g}$ has a normal distribution, so that the probability that the density at a point exceeds a certain value $v_{0}$ is given by

$$
\begin{aligned}
P\left(v_{g}>v_{0}\right) & =\frac{1}{\sqrt{2 \pi}} \int_{v_{0}}^{\infty} e^{-v_{g}^{2} / 2} d v_{g} \\
& =2 \operatorname{erfc}\left(\frac{v_{0}}{\sqrt{2}}\right) .
\end{aligned}
$$

The total overdensity of the combined $t=g+b$ field is given by

$$
\delta t=v_{t} \sigma_{t}=v_{g} \sigma_{g}+v_{b} \sigma_{b} .
$$

We assume that galaxies form only in the regions where $v_{t}$ exceeds the biasing threshold $v_{c}$. This threshold combined with a fixed value $v_{b}$ for the background field yields

$$
v_{0}=v_{c}\left(\frac{\sigma_{t}}{\sigma_{g}}\right)-v_{b}\left(\frac{\sigma_{b}}{\sigma_{g}}\right) .
$$

If we take the asymptotic expansion of erfc $(z)$, the probability for the total density exceeding this threshold $v_{0}$ becomes dependent on $v_{b}$ :

$$
\begin{aligned}
P\left(v_{t}>v_{c}\right) \propto \exp & {\left[-\frac{\left(v_{c} \sigma_{t}-v_{b} \sigma_{b}\right)^{2}}{2 \sigma_{g}^{2}}\right] } \\
& \approx \exp \left(\alpha v_{b}-\frac{1}{2} \beta v_{b}^{2}\right) .
\end{aligned}
$$

Here $\alpha$ and $\beta$ have a simple relation to the threshold and the respective rms amplitudes:

$$
\alpha=v_{c}\left(\frac{\sigma_{t} \sigma_{b}}{\sigma_{g}^{2}}\right) ; \beta=\left(\frac{\sigma_{b}}{\sigma_{g}}\right)^{2}
$$

In BBKS the galaxies are considered as peaks of the density distribution, which makes the details of the calculations more difficult, but still the generic form of the relation (2.4) is very similar - even the $v_{c}$ dependence of $\alpha$ is linear, and $\beta$ is practically constant. Only the numerical coefficients differ a little.

If we associate the threshold for bright galaxy formation (encompassing the major morphological types $\mathrm{Sp}, \mathrm{S} 0$, and $\mathrm{E}$ ) with $v_{c}$ and associate a higher threshold for ellipticals $v_{\mathrm{E}}$, then the elliptical fraction of the galaxies as a function of $v_{b}$ becomes

$$
f_{\mathrm{E}} \propto \exp \left[\left(\alpha_{\mathrm{E}}-\alpha\right) v_{b}-\frac{1}{2}\left(\beta_{\mathrm{E}}-\beta\right) v_{b}^{2}\right] .
$$

This formalism allows us to calculate cumulative distributions; in particular we associate the SO's with an intermediate value of the threshold $v_{\mathrm{so}}$. We can write down the expression for the fraction of E+SO's as

$$
f_{\mathrm{E}+\mathrm{So}} \propto \exp \left[\left(\alpha_{\mathrm{S} 0}-\alpha\right) v_{b}-\frac{1}{2}\left(\beta_{\mathrm{S} 0}-\beta\right) v_{b}^{2}\right] .
$$

The second-order terms in the exponent are negligible, since $\beta$ is practically independent of the thresholds. Therefore the relation between fractional abundances and the linear overdensity of the background is highly nonlinear and exponentially amplified:

$$
\begin{gathered}
f_{\mathrm{E}} \propto \exp \left[\left(\alpha_{\mathrm{E}}-\alpha\right) v_{b}\right] \\
f_{\mathrm{E}+\mathrm{So}} \propto \exp \left[\left(\alpha_{\mathrm{So}}-\alpha\right) v_{b}\right] .
\end{gathered}
$$


This suggests a power-law relation between $f_{\mathrm{E}}$ and $f_{\mathrm{E}+\mathrm{so}}$, where the explicit dependence on the background density has been eliminated. Even the dependence on the particulars of the window function are mild, since these cancel out with the rms values:

$$
\frac{d \log f_{\mathrm{E}}}{d \log f_{\mathrm{E}+\mathrm{SO}}}=\frac{\alpha_{\mathrm{E}}-\alpha}{\alpha_{\mathrm{SO}}-\alpha} \approx \frac{v_{\mathrm{E}}-v_{c}}{v_{\mathrm{SO}}-v_{\mathrm{c}}} .
$$

In principle one could improve this simple model using the peak formalism of BBKS, which should give about the same results. Since here we only emphasize the gross features of the morphology-density relation, we did not feel compelled to go into such complications, since other nonlinear effects most likely dominate.

We adopt the galaxy filter scale $R_{g}=0.375 h^{-1} \mathrm{Mpc}$ taken from BBKS for both the analytic and the $N$-body experiments. Using the BBKS notation, taking $\gamma=0.55$, appropriate for this filter, and choosing $v_{c}=2$, the mean density of the galaxies becomes

$$
n_{g}=0.023 h^{3} \mathrm{Mpc}^{-3} \text {. }
$$

A typical mass of $2.3 \times 10^{11} h^{-1} M_{\odot}$ is associated with a Gaussian filter of this size. To estimate the background mass scale, we take a typical galaxy mass to be about 6 times larger and assume the background region contains 10 times more mass. This yields a filter scale for the background $R_{b}=4 R_{g}$. From Figure 1 of BBKS, one can estimate the relative power within the two filters to give $\sigma_{b} / \sigma_{t} \approx 0.4-0.5$, which translates to $\alpha \approx 0.5-0.6$.

\section{DATA ON MORPHOLOGICAL TYPE-DENSITY CORRELATIONS}

There are three data sets available which describe the dependence of morphological type on local density. Dressler (1980) used projected densities in his study of a sample of 55 rich clusters, and Postman and Geller (1983) measured the mor- phological fraction as a function of local density in the CfA catalog. Giovanelli, Haynes, and Chincarini (1986) use galaxies in the Perseus-Pisces Supercluster and plot morphological fractions as a function of projected overdensity. The variations of morphological fraction found seem to be quite similar, although at first glance projection effects make it difficult to quantitatively compare the two results. All three papers provide the fractional abundances of ellipticals (E), S0's, and spirals (S). The data show quantitatively how the spiral fraction decreases and the elliptical fraction increases in progressively denser environments. However, the SO population is intermediate in its characteristic dependence on environment and shows no simple monotonic trend. The three different fractions are normalized to add up to unity.

Encouraged by the simple analytic relationships suggested in $\S$ II, we replot the data from the samples in a way that is independent of the local density measure, so that the three samples can be intercompared. First, we combine the ellipticals and SO's into a single class whose fraction corresponds to the intermediate threshold $v_{\mathbf{s} 0}$. Second, in order to eliminate the explicit dependence on density, we plot the elliptical fraction $f_{\mathbf{E}}$ (corresponding to the high-threshold $v_{\mathrm{E}}$ ) against the combined $\mathrm{E}+\mathrm{S} 0$ fraction $f_{\mathrm{E}+\mathrm{so}}$. We use only strongly overdense, virialized regions $(\delta \rho / \rho>170)$ to minimize confusion from clumping on intermediate scales at low background density. Also, projection contamination is small in this regime. We do not explicitly make use of the spiral fraction since they provide no further information due to the normalization constraint. Furthermore, since the analytic theory predicts a power-law relation between the respective abundances, we plot $\log f_{\mathrm{E}}$ against $\log f_{\mathrm{E}+\mathrm{so}}$ in Figures $2 a$ and $2 b$.

We see a remarkable concordance between the data sets implying a power-law relation

$$
\frac{d \log f_{\mathrm{E}}}{d \log f_{\mathrm{E}+\mathrm{S} 0}}=1.6 .
$$

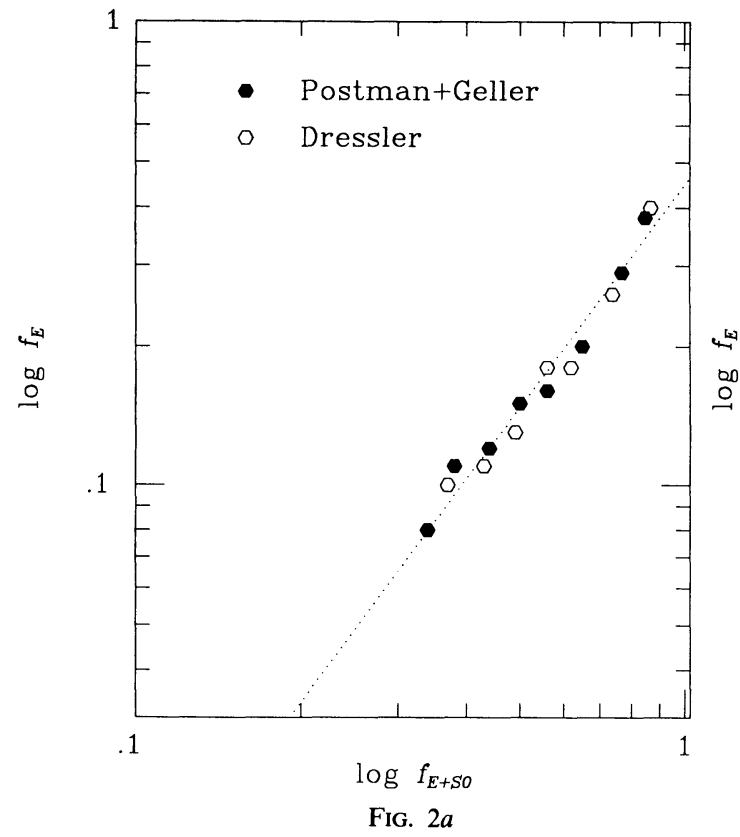

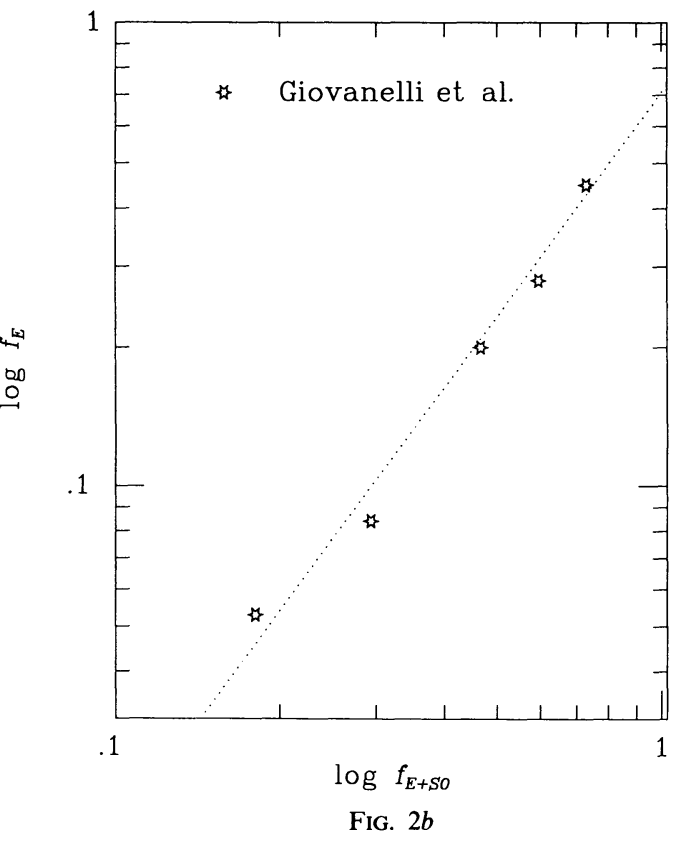

Fig. 2. - The fractional abundance of ellipticals $\left(f_{\mathrm{E}}\right)$ plotted against the combined fraction of ellipticals and S0's $\left(f_{\mathrm{E}+s 0}\right)$. Data from Dressler $(1980)$ and Postman and Geller (1984) are shown in Fig. 2a, while the data of Giovanelli et al. (1986) are shown in Fig. 2b. The dotted line corresponds to a power-law fit with a slope of 1.6 . 


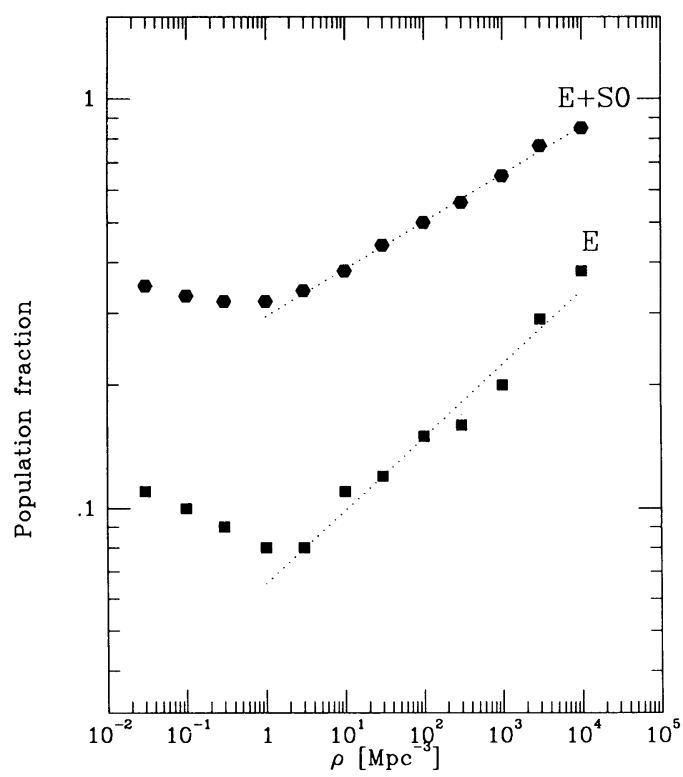

Fig. 3.-The fractions of $E$ and $E+S 0$ galaxies as a function of density in the Postman and Geller sample. In the nonlinear regime above about four galaxies per $\mathrm{Mpc}^{3}$ (corresponding to an overdensity of $\sim 200$ ), the fractions show a power law dependence on local density.

The excellent fit to a power law indicates that projection effects do not play a significant role at these high overdensities. This result supports our model which considers the spiral, S0, and elliptical galaxies as parts of a single underlying population characterized by different thresholds. There is an offset between the Giovanelli, Haynes, and Chincarini (1986) data and the other two samples, due to the selection of galaxies in the UGC Catalog by diameter, which biases the catalog against inclusion of early-type galaxies.

Plotting the Postman and Geller (1984) data on a log-log scale, there is power-law dependence of the galaxy abundances on the background density, shown in Figure 3:

$$
\begin{gathered}
\frac{d \log f_{\mathrm{E}}}{d \log \rho}=0.18 \\
\frac{d \log f_{\mathrm{E}+\mathrm{SO}}}{d \log \rho}=0.12 .
\end{gathered}
$$

\section{MODELING THE NONLINEARITIES}

The fundamental difficulty that we encounter in comparing the above results with the observations centers on how to relate the background density $v_{b}$ of the initial Gaussian process to the highly nonlinear density distribution today. This problem cannot be resolved by applying the two-point correlation function. Rather, it is the very high order moments of the density distribution which generate the six decades of the logarithmic overdensity that is observed. A measure of the overdensity is obtained from the number counts of galaxies in a given cell size. This is clearly related to $P_{N}$, the probability that a given cell contains $N$ galaxies, an integral over the $N$-point correlation function. This distribution is known to be extremely skewed toward high $N$ (Crane and Saslaw 1986; Balian and Schaeffer 1989). In Dressler's (1980) study of galaxy morphology in rich clusters, the number distribution of galaxies over bins of projected density satisfied a lognormal dis- tribution, a conclusion that was even reached by Hubble (1936). Most skewed or non-Gaussian distributions can in fact be fitted by a lognormal distribution, and this result should be considered as providing a phenomenological description of the nonlinear galaxy distribution.

Using such a lognormal fit, one can clearly map a nonGaussian distribution into a Gaussian, but this procedure does not guarantee that any spatial coherence will be retained. Dynamical mixing during nonlinear collapse can significantly rearrange the galaxies. However, the success of biased CDM and the density peak prescription as outlined in BBKS suggests that the mapping of initial (Lagrangian) overdensity to final density is a reasonably robust procedure in the absence of dissipation: one would expect that statistically the points at the highest linear overdensities would end up in high-density regions after significant nonlinear evolution has taken place. Conservation of mass enables us to establish a mapping between these two distributions, by requiring that the same mass fraction be associated with the respective overdensity level surfaces. However, this still does not guarantee that this mapping is true in the local sense. The validity of this assumption has recently been investigated by Weinberg (1989), who used the above procedure to obtain from galaxy catalogs Gaussian initial conditions for an $N$-body simulation that was subsequently evolved to be similar to the original galaxy distribution. We further explore this mapping in the $N$-body experiments presented in the next section.

Already the above arguments of lognormality point to a roughly exponential relation between $v_{b}$ and $\rho$, but we decided to adopt an even more phenomenological approach. We use the original data of Postman and Geller (1984), comprising the number of galaxies in the CfA Catalog above a certain local overdensity threshold. We convert these cumulative numbers into fractions, then convert these fractions into a normal Gaussian distribution, by assigning a Gaussian variable $v$ that has the same cumulative fraction associated with it. The relation between the nonlinear overdensity contrast and the Gaussian is surprisingly well fitted by an exponential (Fig. 4):

$$
\delta \rho / \rho=\exp (3.36 v) .
$$

This overdensity dependence of the luminous matter comprises two independent factors. One is the nonlinear gravitational clumping of all mass, including the dark matter. The second is the systematic segregation of the luminous matter from the dark matter. The latter may be expected to have an exponential dependence on $v$ from equation (2.4), as long as the statistical quantity $v$ is providing a reasonable estimate of the physical quantity $v_{b}$. This is addressed with the $N$-body models of $\S \mathrm{V}$.

Since both measurable quantities, the morphological fraction and the local density, depend on the background level in an exponential manner, we expect a generic power law relation between our observables. This should break down at sufficiently low densities, where noise from small-scale clumpiness and projection effects contaminate the measure of local density. At overdensities above 170, virialization will erase substructure. Thus we would expect a flattening of morphological fraction dependence on density below $\sim 1 \mathrm{Mpc}^{-3}$.

\section{N-BODY EXPERIMENTS}

To fully explore the effects of nonlinearities inherent in the gravitational clustering process, we have performed a set of numerical simulations. Initial fluctuations in $L=16 h^{-1} \mathrm{Mpc}$ 


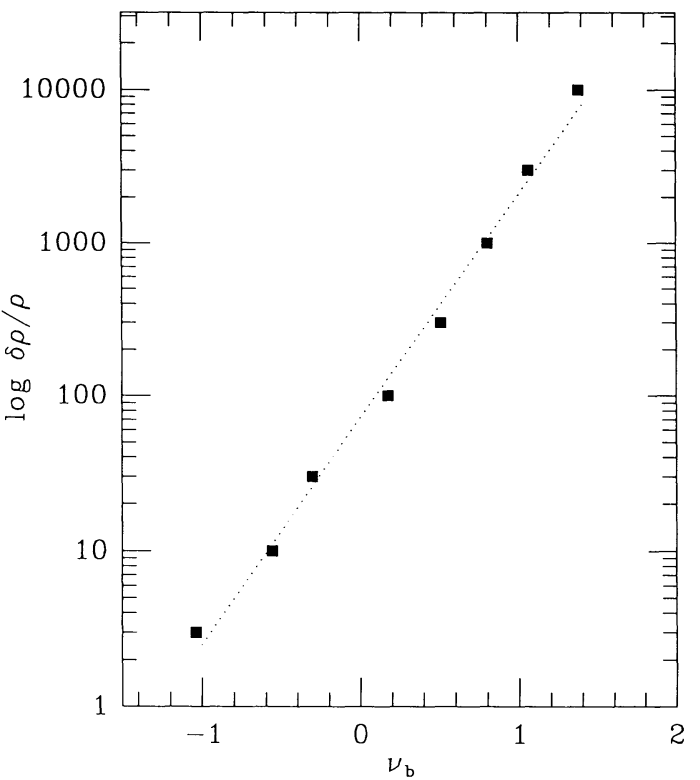

Fig. 4.-The cumulative "filling factor" of galaxies as a function of overdensity $\delta$ in the Postman and Geller sample expressed in terms of an equivalent complimentary error function variable $v$. That is, the fraction of galaxies in the sample whose local density contrast is $\geq \delta$ is given by erfc $(v)$.

periodic cubes were generated on a $N=64$ cubic mesh using Bertschinger's (1987) path integral method over a range of constrained density fields. The constraint used is that the largescale density field filtered with a Gaussian window on a scale $R_{\mathrm{LS}}=0.2 L=3.2 h^{-1} \mathrm{Mpc}$ equal an input value $\delta_{\mathrm{LS}}$. We chose five values of $\delta_{\mathrm{LS}}$ corresponding to $-4 \sigma,-2 \sigma, 0,2 \sigma$, and $4 \sigma$ fluctuations on the filter mass scale $M_{\mathrm{LS}} \sim 1.4 \times 10^{14} M_{\odot}$. The constrained fields are a convenience which allows efficient sampling of rare, high-density environments. The choice of filter scale is, to some degree, arbitrary; the value $M_{\mathrm{LS}} \simeq 10^{14} M_{\odot}$ reflects the mass scale associated with clusters of galaxies. Two realizations are generated for each of the above values of $\delta_{\mathrm{LS}}$, corresponding to the real and imaginary parts of the initial complex density field, yielding a total of 10 models. The initial displacement fields are evolved to an initial redshift $z_{i}=9$ with the Zel'dovich approximation. These are then assigned to a reduced $32^{3}$ particle distribution and the system followed to the present with the P3M N-body code (Efstathiou and Eastwood 1981). The initial conditions and simulations are summarized in Table 1.

Galaxies in this model are assumed to form at the locations of peaks in the initial, filtered density field which lie above

TABLE 1

Simulation Parameters

\begin{tabular}{|c|c|}
\hline Parameter & Value \\
\hline Comoving cube length & $16 h^{-1} \mathrm{Mpc}$ \\
\hline Fourier grid size ....... & $0.25 h^{-1} \mathrm{Mpc}$ \\
\hline Number of particles & 32,768 \\
\hline Gravitational softening & $50 h^{-1} \mathrm{kpc}$ \\
\hline Particle mass ................ & $3.4 \times 10^{10} h^{-1} M_{\odot}$ \\
\hline Total mass . ............... & $1.2 \times 10^{15} h^{-1} M_{\odot}$ \\
\hline Galaxy filter radius ........... & $0.375 h^{-1} \mathrm{Mpc}$ \\
\hline L-S background filter radius $\ldots \ldots$ & $3.2 h^{-1} \mathrm{Mpc}$ \\
\hline Constrained L-S perturbations ................ & $v_{\mathrm{LS}}=4,2,0,-2,-4$ \\
\hline
\end{tabular}

some critical threshold $v_{c}$. To locate the Lagrangian birthplaces of galaxies in the simulations, the initial $64^{3}$ density fields are filtered on a scale $R_{g}=0.375 h^{-1} \mathrm{Mpc}$. Peaks, defined simply as a cell whose density is greater than those of its adjacent 26 neighbors, are located within the realizations, and the nearest locations in the $32^{3}$ particle distribution are "tagged" with the appropriate peak height $v_{g}$. The cumulative abundance of galaxy-scale peaks found this way in the models is in good agreement with the analytic BBKS expectation.

If galaxies behave as collisionless objects after their dissipative formation, then it is appropriate to use the trajectories of the particles tagged at locations of peaks above the $v_{c}$ threshold as tracers of "galaxy" orbits. Figure 5 shows the dynamical evolution inside a comoving $8 h^{-1} \mathrm{Mpc}$ cubic region in one of the $4 \sigma$ constrained background runs. The evolution proceeds in rows from top to bottom. The left-hand column shows particles representing peaks above an elliptical threshold taken to be $v_{\mathrm{E}}=3$. The next column shows peaks associated with the disk (SO and Sp) population - those above the critical threshold for bright galaxy formation $v_{c}$ but below the spheroid limit $v_{\mathrm{E}}$. We assume a value of $v_{c}=2$. The subsequent column shows all those peaks below $v_{c}$ ("failed" protogalaxies?), and the right-most column shows the general mass distribution.

Higher peaks are more strongly clustered in the initial, linear field as expected. By a redshift $z \simeq 1$, the elliptical population is already concentrated in the dense core of the collapsing protocluster. The disk population inhabits the surrounding, lower density environments. At $z=0$, there are $\sim 90$ galaxies (particles associated with peaks above $v_{c}$ ) within an Abell radius of the cluster center-this would correspond to Abell richness class II. The velocity dispersion of $\sigma \simeq 700 \mathrm{~km} \mathrm{~s}^{-1}$ is not atypical of a richness II cluster.

We can perform straightforward morphology-density measurements on the numerical experiments by using projected snapshots of the modeled galaxy distribution down each of the simulation axes. Local two-dimensional densities are determined by Dressler's (1980) criterion based on the distance to the 10th nearest galaxy. Type fractions are computed from the ensemble of projections for each box, and results from the independent volumes combined to improve statistics. Counts of spheroid (E) and disk (S0 and $\mathrm{Sp}$ ) galaxies found using the peak threshold criteria are given for all the models in Table 2. Values of $v_{\mathrm{E}}=3$ and $v_{c}=2$ and assumed, and the S0 counts assume an intermediate threshold $v_{\text {so }}=2.5$.

Figure 6 shows the resulting elliptical and $\mathrm{E}+\mathrm{S} 0$ fractions versus projected density determined from stacking the three

TABLE 2

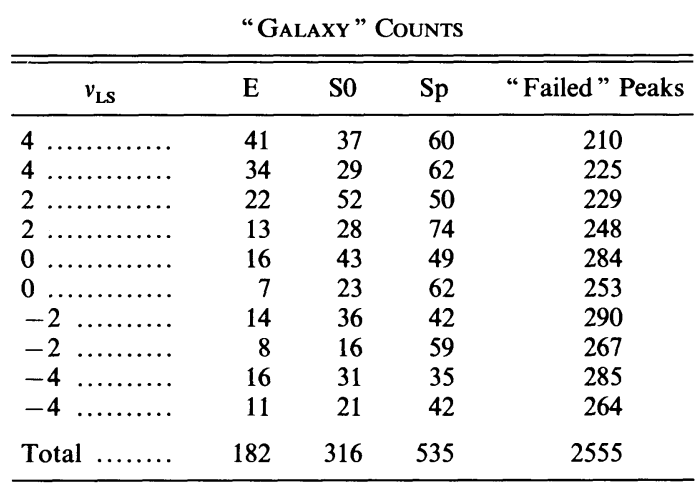




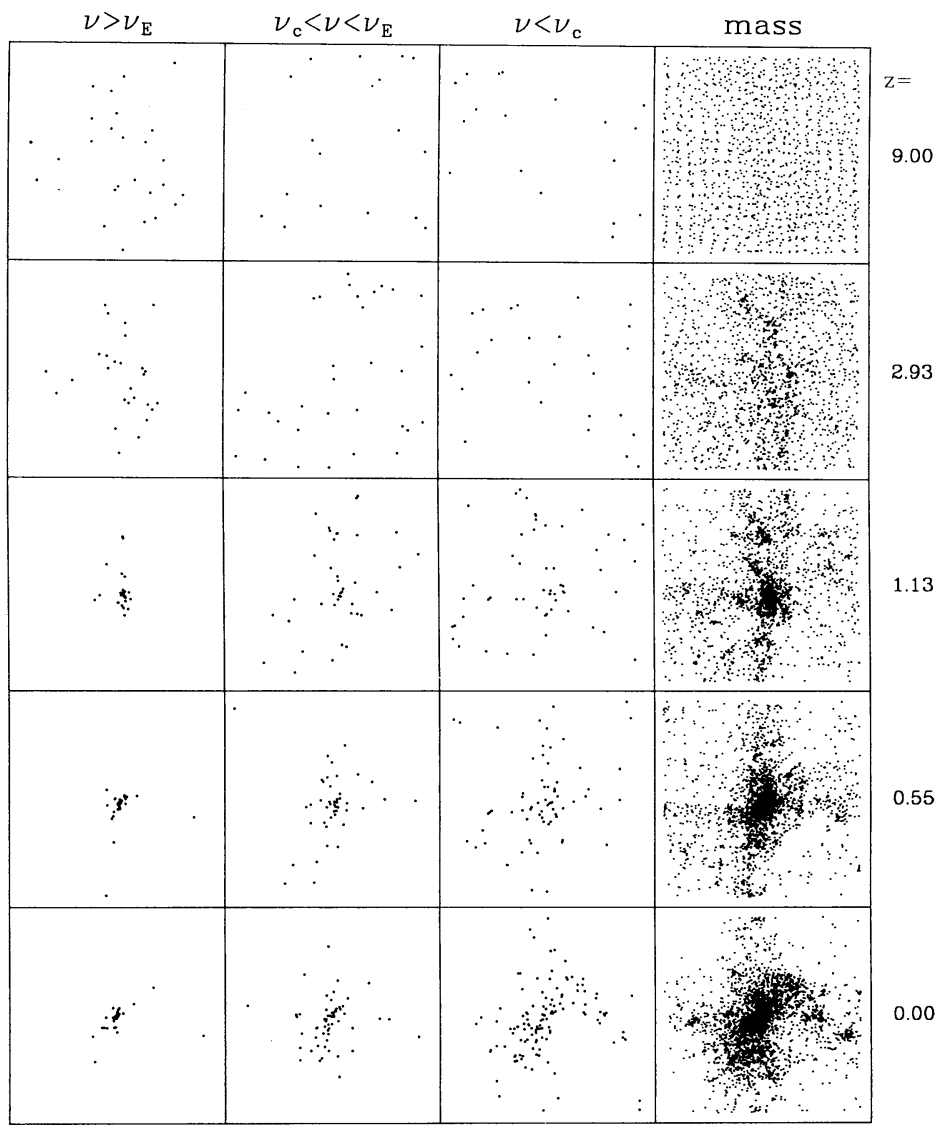

Fig. 5.-Evolution within a comoving $8 h^{-1} \mathrm{Mpc}$ cubic region on one of the simulations constrained to produce a moderately rich cluster by the present epoch. Particles associated with peaks above the elliptical threshold $v_{\mathrm{E}}$ are more clustered than those between $v_{c}$ and $v_{\mathrm{E}}$ which are in turn more clustered than the total mass distribution.

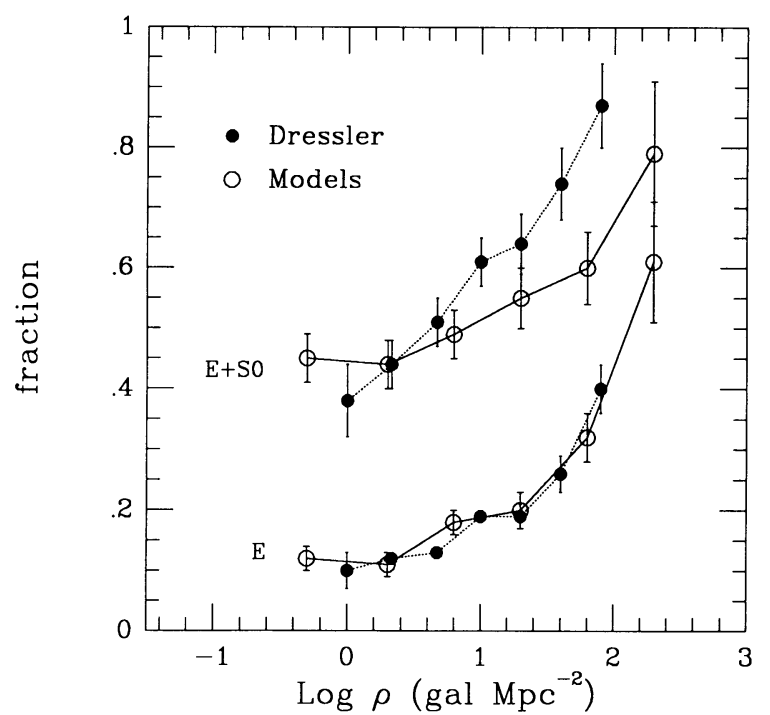

FIG. 6.-The morphology-density relation for $\mathrm{E}$ and $\mathrm{E}+\mathrm{S} 0$ galaxies for both the Dressler sample (open circles) and the ensemble of numerical experiments ( filled circles) determined using $h=0.5$. The simulations assume $v_{c}=2$, $v_{\mathrm{S} 0}=2.5$ and $v_{\mathrm{E}}=3$. The observed behavior of the elliptical population is reproduced well by the experiments. Results for the combined E+SO population are not as compelling. orthogonal projections of all 10 experiments. Error bars are $1 \sigma$ deviations within each bin. Dressler's data are the solid circles. The simulations reproduce the Dressler data for the elliptical fraction versus density extremely well. The combined $\mathrm{E}+\mathrm{S} 0$ behavior is in poorer agreement. There are not enough SO's in the highest density environments, even with the low value of $v_{\mathrm{So}}=2.5$. Figure 7 shows the elliptical versus $\mathrm{E}+\mathrm{S} 0$ fractions from the simulations. The data are in fairly good agreement with the theoretically expected power law of slope

$$
\frac{v_{\mathrm{E}}-v_{c}}{v_{\mathrm{SO}}-v_{c}}=2
$$

shown as the dashed line and slightly steeper than the observed slope taken from Figure $2 a$. Attempts at increasing the S0 fraction at high densities by lowering $v_{\text {so }}$ fail because this simultaneously generates more S0's in low density environments than are observed. The discrepancy between the analytic and numerical results for the S0's may be due to subtle effects on the tail of the peak height distribution arising from the discrete nature of the simulations; the modulation is very sensitive to the local shape of the peak height distribution. For the adopted filter scale, the appropriate value of $v_{c}$ which reproduces the observed mean density of bright galaxies is $\sim 2.3$ (BBKS). Had we chosen this value of $v_{c}$ and shifted to $v_{\mathrm{E}}=3.2$ in order to produce the correct global abundance of ellipticals, the number of peaks above $v_{E}$ in the simulations would have been reduced by a factor of 2 and the statistics therefore much 


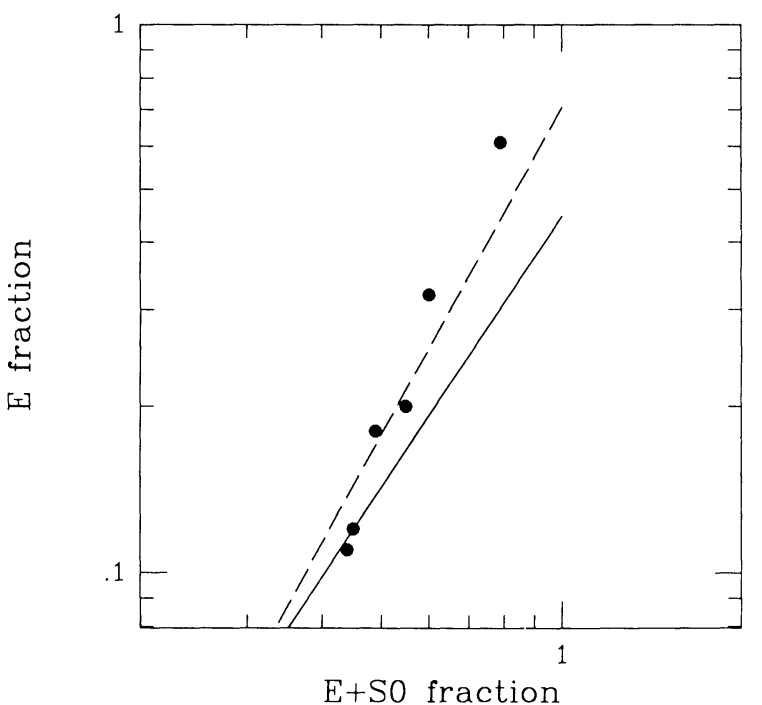

Fig. 7.-The $\mathrm{E}$ fraction is plotted against combined $\mathrm{E}+\mathrm{S} 0$ fraction for the numerical experiments (solid circles). The dashed line shows the slope of 2 expected from the analytic model, while the solid line is the observed slope taken from Fig. $2 a$.

poorer. The set of higher values for the thresholds imply a steeper local slope, thus a steeper modulation for the E+S0 fraction, a shift in the right direction. The simulations provide support for a peak height distinction between disk and spheroid morphologies, but a finer distinction between S0 and Sp galaxies based on this simple threshold picture is not entirely clear.

One can also use the numerical experiments to investigate aspects of the non-linear analytic model presented in the preceding section. Figure 8 shows the local galaxy density contrast $\delta$ plotted against fraction of galaxies expressed in terms of the complimentary error function variable $v$ as in $\S$ IV). A three dimensional density based on distance to the 10th nearest neighbor is used. For collapsed regions with $\log \delta \gtrsim 2$, the

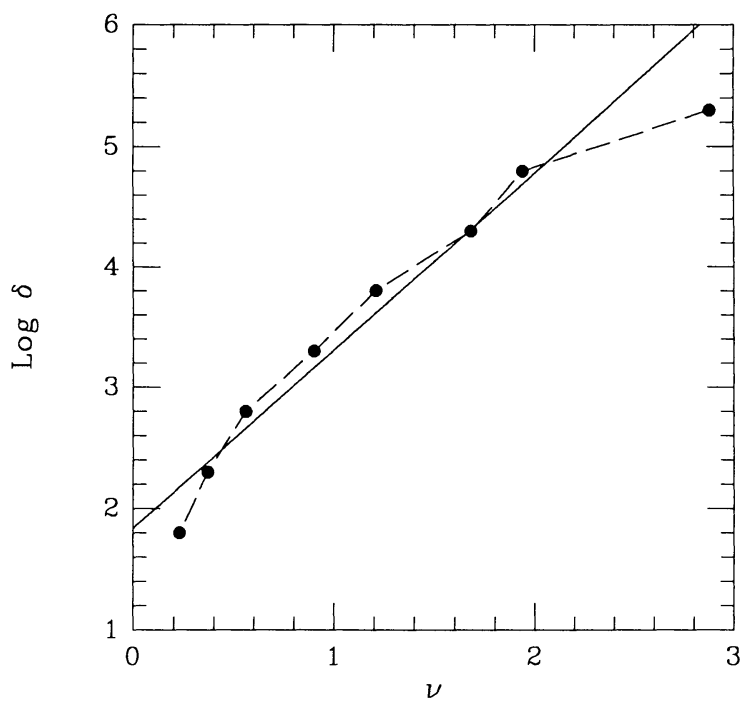

FIG. 8.- The cumulative "filling factor" for galaxies in the simulations (dashed line) along with the observed relation (solid line) taken from Fig. 4. The experiments show a roughly lognormal mapping in the nonlinear regime similar to that seen observationally.

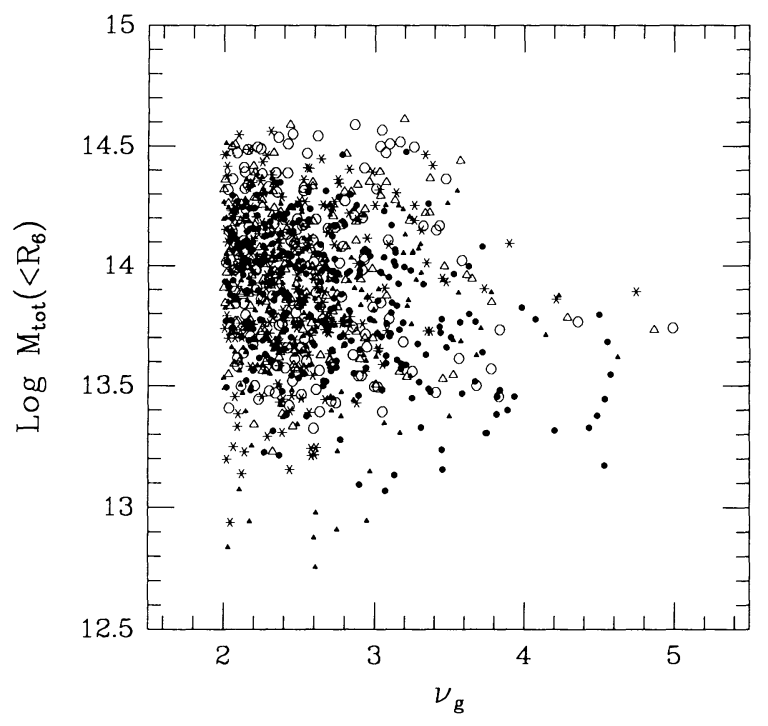

Fig. 9.- The total mass (assuming $h=0.5$ ) contained within a radius defined by the (three-dimensional) distance to the sixth nearest galaxy in the final, nonlinear galaxy distribution is plotted against initial peak height $v_{g}$ filtered on the galaxy mass scale. A large scatter in this "background" mass scale is evident - the median value is roughly 200 times the employed galaxy filter mass. Different symbol types refer to different values of the initial LS background constraint: filled circles, $v_{\mathrm{LS}}=4$; filled triangles, $v_{\mathrm{LS}}=2$; asterisks, $v_{\mathrm{LS}}=0 ;$ open triangles, $v_{\mathrm{LS}}=-2 ;$ and open circles, $v_{\mathrm{LS}}=-4$.

simulated data follow an exponential mapping similar to that seen in Figure 4 for the observational data.

Some of the difficulties one will encounter when attempting analytic calculations based on a peak-background split assumption can be appreciated by examining Figure 9. Here the total mass within a sphere of radius $R_{6}$, the distance to the sixth nearest galaxy, is plotted against peak height on galactic scales. Interpreting this mass as the "background" mass scale for analytic calculation, we see that there is a rather large range, about a factor 30 , in the true local background mass. This makes definition of an appropriate, single background mass scale problematic. The median value of $M\left(<R_{6}\right)=10^{14}$ $M_{\odot}$ is a factor of 200 greater than the filter mass of the galaxy scale.

Does the background density field filtered on this scale contain any useful information regarding nonlinear galaxy densities? Figure 10 shows the final local galaxy densities based on $R_{6}$ plotted against the background perturbation height $v_{b}$ of the density field filtered using a Gaussian $M_{b}=$ $10^{14} M_{\odot}$. A clear monotonic trend is evident, but the scatter in density at a fixed background value can be quite large. This is especially true in the transition region separating the linear and nonlinear regimes.

We note that the local galaxy density is, as expected, a biased estimate of the mass density measured within the same volume, as illustrated in Figure 11. The local mass overdensity $\delta_{\rho}$ is related to the galaxy overdensity $\delta_{g}$ by a power law over six orders of magnitude

$$
\delta_{\rho} \propto \delta_{g}^{0.8}
$$

We stress that the scale at which the local mass density is measured is defined by the galaxy distribution. The above relation is not defined at a fixed background mass scale; rather, it holds over the range of background masses illustrated in 


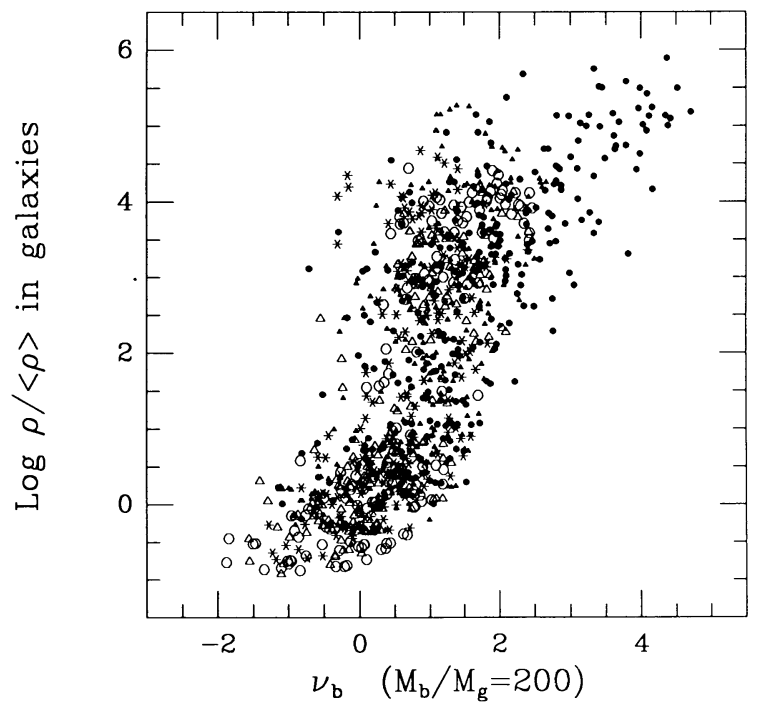

FIG. 10.-The final galaxy density based on the three-dimensional distance to the 6th nearest galaxy plotted against the initial perturbation height $v_{b}$ filtered on the median background mass scale of Fig. 9. Symbol types are the same as in Fig. 9. The final nonlinear density is correlated with the linear background density, but a large scatter is evident.

Figure 9. Because of the difficulty in estimating dynamical masses, this relation will be hard to test observationally. However, density reconstruction techniques such as that developed by Bertschinger and Dekel (1989) may provide a means of measuring this relation in the linear to mildly nonlinear regime $\delta_{\rho} \lesssim 10$.

\section{DISCUSSION AND CONCLUSIONS}

We have presented evidence that the observed galaxy morphology-density relation may be intrinsic to the primordial fluctuations from which the galaxies formed. Following Faber's (1982) suggestion of a relation between morphology and height of initial density perturbation, we adopt a threshold criterion defining morphological type in the biased CDM scenario. Using a simplified, fully analytic version of the BBKS, formalism we have shown how a background cluster or group of galaxies may modulate the relative abundances of galaxies with different Hubble types.

By combining the observational data on the density dependence of ellipticals and S0's, we have also found a tight powerlaw correlation between $f_{\mathrm{E}}$ and $f_{\mathrm{E}+\mathrm{so}_{0}}$. This argues that ellipticals and S0's constitute a single-parameter family with respect to the density dependence. Despite the fact that nonlinear evolution introduces a strongly non-Gaussian tail into the galaxy distribution, we have found that the logarithmic

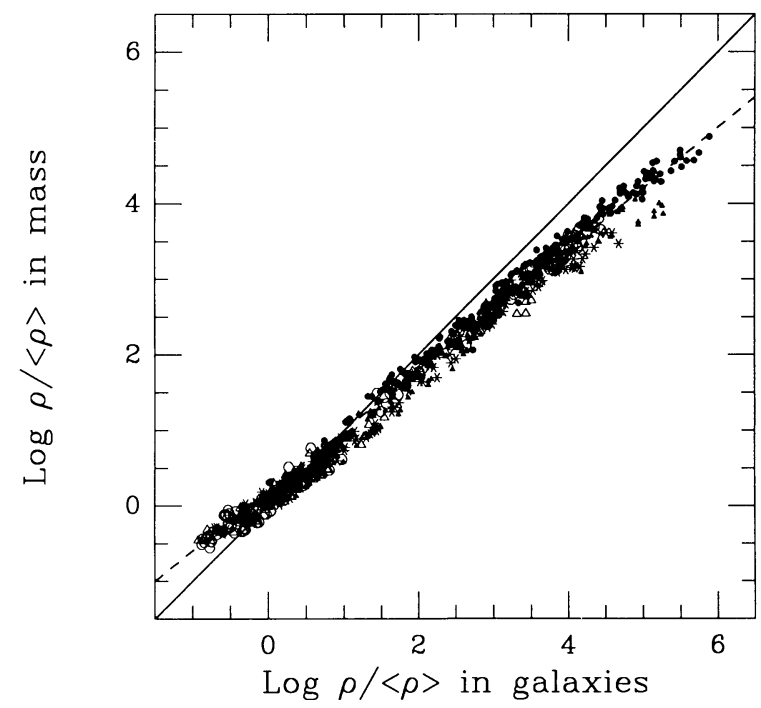

FIG. 11.-Relation between the local galaxy and mass density measured within a sphere of radius $R_{6}$, the distance to the sixth nearest galaxy. The data follow a power law of slope 0.8 (dashed line).

overdensity of the galaxies in the CfA catalog is extremely well fitted by a Gaussian distribution. Furthermore, both $f_{\mathrm{E}}$ and $f_{\mathrm{E}+\mathrm{so}}$ have a power-law dependence on the local overdensity. These observed trends are of intrinsic interest and independent of any assumptions about CDM or a specific model for galaxy formation.

We have extended the analytic approach by performing $N$-body simulations with 32,768 mass points in comoving volumes of dimensions $16 h^{-1} \mathrm{Mpc}$. The results agree with the observed relation between elliptical fraction and local galaxy density. However, in contrast to the analytic work, the simulations do not reproduce as well the observed behavior of the $\mathrm{E}+\mathrm{S} 0$ population. We have discovered and quantified an interesting trend in the simulations between the total mass and galaxy densities: a power-law relation of the form $\delta_{\rho} \propto \delta_{g}^{0.8}$ is apparent over a range of six orders of magnitude in galaxy density. We anticipate that future developments in density reconstruction techniques and other dynamical mass estimation methods may allow this prediction to be tested over a modest dynamic range in density.

We would like to acknowledge useful conversations with Rosie Wyse. We are indebted to M. Postman and M. Geller for allowing us to use some of the raw data related to their original publication. This research has been supported by the Miller Foundation for Basic Research in Science and by NSF grant AST-8819802 in Berkeley, by an NSF-Hungary exchange grant, and by OTKA in Hungary.
Abell, G. O. 1958, Astr. Ap. J. Suppl., 50, 241.

Balian, R., and Schaeffer, R. 1989, Astr. Ap., 200, 1.

Bardeen, J. M., Bond, J. R., Kaiser, N., and Szalay, A. S. 1986, Ap. J., 304, 15 (BBKS).

Bertschinger, E. 1987, Ap. J. (Letters), 323, L103.

Bertschinger, E., and Dekel, A. 1989, Ap. J. (Letters), 336, L5.

Blumenthal, G. R., Faber, S. M., Primack, J. R., and Rees, M. J. 1984, Nature, 311, 517 .

\section{REFERENCES}

Crane, P., and Saslaw, W. 1986, Ap. J., 301, 1

Davis, M., and Geller, M. J. 1976, $A p . J ., 208,13$.

Dekel, A., and Silk, J. 1986, Ap. J., 303, 39.

Dressler, A. 1980, Ap. J., 236, 351.

Efstathiou, G. P., and Eastwood, J. W. 1981, M.N.R.A.S., 194, 503.

Evrard, A. E. 1989, Ap. J., 341, 26.

Faber, S. M. 1982, in Astrophysical Cosmology, ed. H. A. Bruck, G. V. Coyne and M. S. Longair (Vatican City: Pontifica Academia Scientiarum), p. 191. 
Giovanelli, R., Haynes, M. P., and Chincarini, G. L. 1986, Ap. J., 300, 77

Hubble, E. P. 1936, The Realm of the Nebulae (New Haven: Yale University

I. Press), Fig. 7.

' Morgan, W. W. 1961, Proc. Nat. Acad. Sci., 47, 905.

I.I Postman, M., and Geller, M. J. 1984, Ap.J., 281, 95.

Schweizer, F. 1989, in Dynamics and Interactions of Galaxies, ed. R. Wielen (Springer-Verlag: Heidelberg), in press.

Toomre, A., and Toomre, J. 1972, Ap. J., 178, 623.

Weinberg, D. 1989, Ph.D. thesis, Princeton University.

A. Evrard: Department of Physics, 1049 Randall Lab, University of Michigan, Ann Arbor, MI 48109

J. SILK : Department of Astronomy, University of California, Berkeley, CA 94720

A. S. Szalay: Department of Physics and Astronomy, The Johns Hopkins University, Baltimore, MD 21218 